\title{
The influence of membranous stretched urethral length and urethral circumference on postoperative recovery of continence after radical prostatectomy: A pilot study
}

\author{
Dae Sung Cho, MD; ${ }^{*}$ Eun Ju Lee, MD; ${ }^{\dagger}$ Se Joong Kim, MD; Sun II Kim, MD
}

*Department of Urology, Bundang Jesaeng General Hospital, Bundang-gu, Seongnam-si, Gyeonggi-do, South Korea; †Department of Radiology, Ajou University School of Medicine, Yeongtong-gu, Suwon-si, Gyeonggi-do, South Korea; §Department of Urology, Ajou University School of Medicine, Yeongtong-gu, Suwon-si, Gyeonggi-do, South Korea

Cite as: Can Urol Assoc J 2015;9(5-6):E262-6. http://dx.doi.org/10.5489/cuaj.2563

Published online May 13, 2015.

\section{Abstract}

Introduction: We investigate the influence of stretched membranous urethral length (SUL) and urethral circumference (UC) on postoperative recovery of continence after radical prostatectomy (RP). Methods: To evaluate the distal continence zone intraoperatively, we individually measured and recorded stretched membranous urethral length (distance between the urogenital diaphragm and the prostate apex with cephalad retraction, SUL) and urethral circumference (UC) after exposure of the urethra. We analyzed the association between magnetic resonance imaging-measured membranous urethral length (MRIL) and urethral diameter (MRID) and intraoperative SUL and UC and influence on return to continence. Results: The mean patient age, SUL and UC were $66.5 \pm 6.0$ years, $24.2 \pm 3.3 \mathrm{~mm}$, and $27.5 \pm 4.4 \mathrm{~mm}$, respectively. MRIL and MRID were $11.3 \pm 1.6 \mathrm{~mm}$ and $10.6 \pm 1.9 \mathrm{~mm}$, respectively. In the bivariate correlation analysis, there was no statistically significant correlation between SUL and MRIL $(p=0.201)$ and between UC and MRID $(p=0.124)$. In the Kaplan-Meier curve analysis, cumulative continence rates between the two groups dichotomized at the median value according to age $(p=0.0519)$, SUL $(p=0.6583)$, UC $(p=0.4031)$, MRIL $(p=0.4042)$, and MRID $(p=0.8191)$ were not significantly different. High SUL-to-MRIL ratio $(>2.2)$ was the only significant predictor of lower cumulative continence rate $(p=0.0457)$.

Conclusions: MRIL measured during surgery was not associated with postoperative continence recovery after RP. We observed that an excessively long membranous urethra compared to the urethral length on preoperative MRI is predictive of poorer postoperative continence recovery. However, small sample size and potential confounding surgical factors limit the significance of this study.

\section{Introduction}

Urinary continence is one of the most important quality of life factors and a major drawback in patients after radical prostatectomy (RP). The reported percentages of patients with urinary incontinence after RP range from $6 \%$ to $20 \% .^{1-3}$ However, improvements in surgical techniques and advances in the knowledge of pelvic and perineal anatomy have led to better preservation of continence and potency after RP. ${ }^{4}$

Several factors have been proposed to affect male continence recovery after RP often with conflicting evidence. These include perioperative factors, such as age and prostate volume, ${ }^{5}$ and certain anatomic or surgical factors important in continence preservation (injury to the arterial supply and preservation of urethral support mechanisms, including the endopelvic fascia and puboprostatic ligaments). ${ }^{6,7}$

Magnetic resonance imaging $(\mathrm{MRI})$ is the best method to evaluate clinical stage, prostatic anatomy and measurements, and urethral lengths before RP. ${ }^{8}$ Few studies, however, have assessed intraoperative prostatic and urethral measurements, how they are associated with preoperative MRI measurements, and whether they can predict continence outcomes. ${ }^{9}$ Borin and colleagues ${ }^{10}$ reported that more aggressive apical dissection with transaction of the striated external sphincter resulted in no measurable change in time to continence or overall continence.

The purpose of this study was to investigate the influence of stretched membranous urethral length (SUL) and urethral circumference (UC) on postoperative recovery of continence after RP. 


\section{Methods}

\section{Patient characteristics}

This prospective study, which was approved by Ajou University Hospital Institutional Review Board, included 48 patients undergoing open RP, as performed by a single surgeon (SIK), for newly diagnosed localized prostate cancer from December 2009 to November 2011 and for whom preoperative MRI images of the prostate were available. Seven of these patients were excluded from our study because of urgency or urge incontinence before surgery, and 5 were excluded because they had a history of diabetes mellitus and the possibility of neurogenic bladder. Nine patients with inadequate urethral measurement during operation were also excluded. As a result, a total of 27 patients were enrolled in this prospective study.

\section{MRI technique}

All MRI studies were performed on a 3 Tesla whole-body magnetic resonance scanner (Intera Achieva, Philips Medical Systems, the Netherlands) using a pelvic phasedarray coil. Examinations included multiplanar turbo spinecho T2-weighted images (T2WI), axial turbo spin-echo T1-weighted images, axial single-shot echo-planar diffusionweighted imaging, and dynamic contrast-enhanced (DCE) MRI using a three-dimensional (3D) fat-suppressed spoiled gradient-echo T1-weighted sequence.

\section{MRI interpretation}

The attending radiologist (EJL), blind to the patient's clinical and pathological data, measured and recorded the membranous urethral length (MRIL) and the urethral diameter
(MRID) from the preoperative MRI scan. MRIL and MRID were measured on T2-weighted MR images. A straight line between the prostatic apex and the penile bulb was drawn in the mid-sagittal plane and the coronal plane, and their mean value was used as MRIL. Similarly, anteroposterior and horizontal lines were drawn, each connecting opposite ends of the urethral margins and crossing the middle of the lumen in the axial plane just below the prostatic apex, and their mean value was used as MRID (Fig. 1).

\section{Surgical technique}

In brief, the endopelvic fascia was opened on both sides of the prostate and the levator ani muscle fibers were teased off the prostate surface. After cutting the puboprostatic ligament, the deep dorsal vein was suture-ligated and cut without suspending it to the pubic bone and the urethra was exposed. Then, a right-angled clamp was passed beneath the urethra, and with a cephalad retraction being applied, the distance between the urogenital diaphragm and the prostatic apex (stretched membranous urethral length, SUL) was measured with a measuring tape (Fig. 2). Then, the measuring tape was passed beneath the urethra and the UC was measured with the urethral catheter out of place (Fig. 3). The urethra was cut $2 \mathrm{~mm}$ distal from the prostatic apex. Whenever, extracapsular tumour extension was suspected, the neurovascular bundle on that side was widely cut. Otherwise, the levator fascia was opened and gently released to save the neurovascular bundle. Apical, posterior and lateral dissection of the prostate and dissection of seminal vesicles and vas deferens was carried out in a usual fashion. After dividing the bladder neck, reconstruction was performed if needed using full-thickness interrupted sutures. Neither bladder neck intussusceptions, nor posterior reconstruction was attempted. Urethrovesical anastomosis was performed by placing sutures at 12-, 2-, 5-, 6-, 7- and 10-o'clock

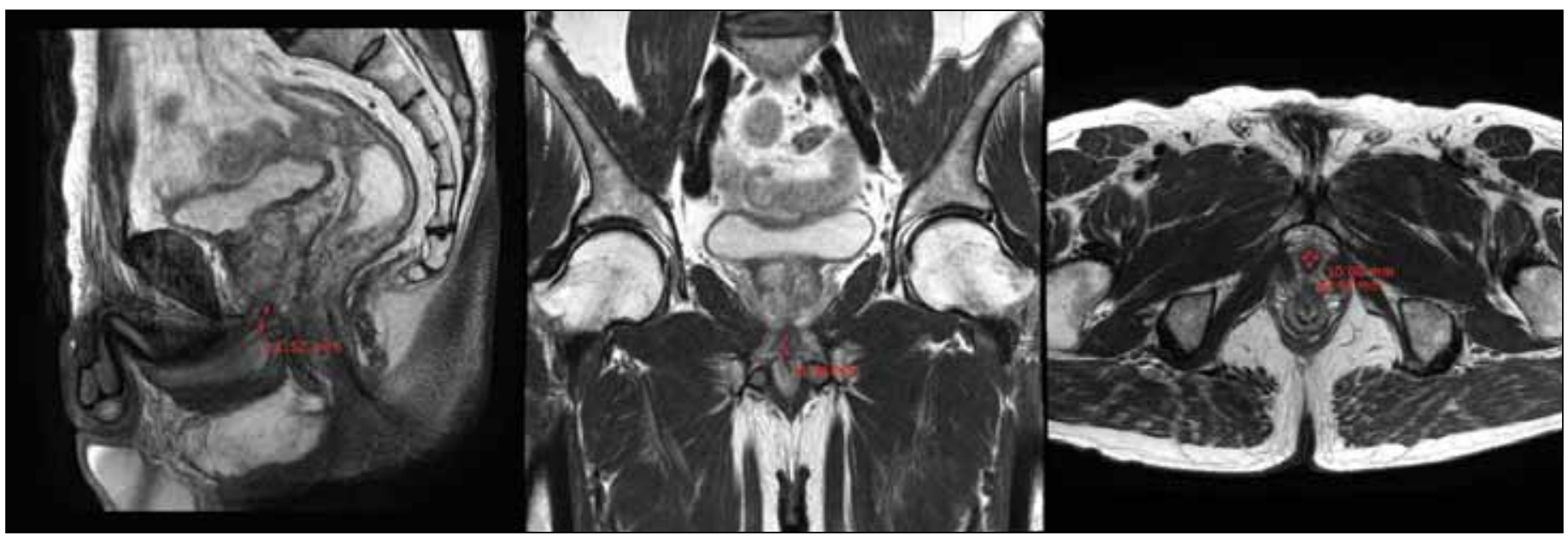

Fig. 1. Measurement of membranous urethral length (MRIL) and urethral diameter (MRID) on T2 weighted images of magnetic resonance imaging. 


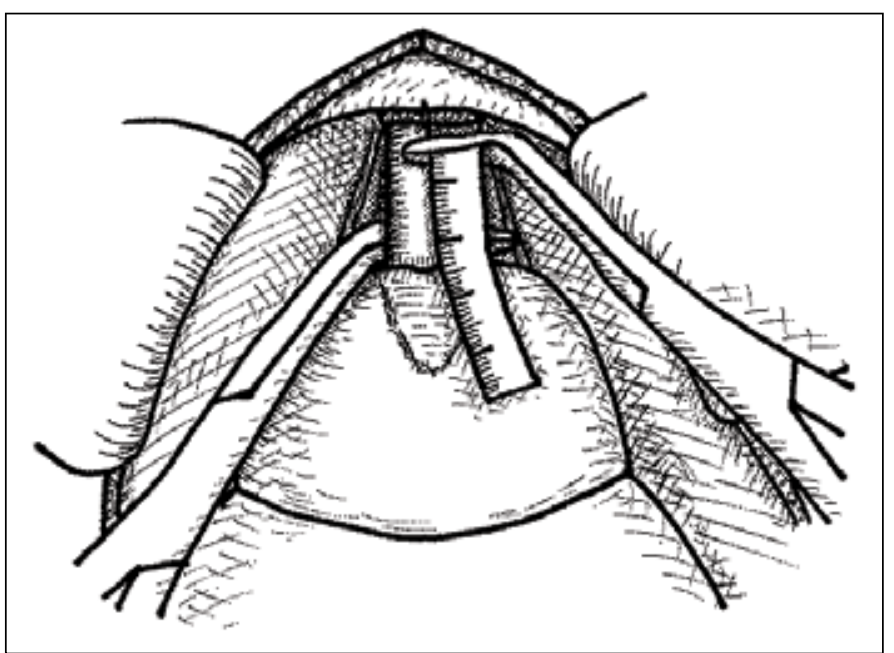

Fig. 2. Illustration of intraoperative measurement of stretched membranous urethral length (SUL).

positions. At 1, 3, 6, 9, and 12 months after the operation, patients were asked to complete a modified International Prostate Symptom Score questionnaire supplemented with a last question inquiring the number of daily pad use ranging from 0 to 3 or more. The time to continence (TTC) was defined as the time the patient first became pad-free.

\section{Statistical analysis}

The bivariate correlation analysis was used to determine the relationship between SUL and MRIL, and between UC and MRID. Age, SUL, UC, MRIL, MRID and SUL to MRIL ratio were dichotomized at the median value. The cumulative continence rate was calculated using the Kaplan-Meier method and the comparison between groups was made using the log-rank test. For all tests, $p<0.05$ indicated statistical significance.

\section{Results}

All 27 enrolled patients were followed postoperatively for at least 1 year. At year 1, 6 patients (22.2\%) were using one or more pads a day (Table 1 ).

The mean patient age, SUL and UC were $66.5 \pm 6.0$ years, $24.2 \pm 3.3 \mathrm{~mm}$, and $27.5 \pm 4.4 \mathrm{~mm}$, respectively. MRIL and MRID were $11.3 \pm 1.6 \mathrm{~mm}$ and $10.6 \pm 1.9 \mathrm{~mm}$, respectively. In the bivariate correlation analysis, there was no statistically significant correlation between SUL and MRIL, and between UC and MRID ( $p=0.201, p=0.124)$. In the Kaplan-Meier curve analysis, cumulative continence rates between the two groups dichotomized at the median value according to age $(p=0.0519), \operatorname{SUL}(p=0.6583), \cup C(p=0.4031)$, MRIL $(p=0.4042)$, and MRID $(p=0.8191)$ were not significantly different. High SUL-to-MRIL ratio $(>2.2)$ was the only significant predictor of lower cumulative continence rate $(p=0.0457$, Fig. 4$)$.

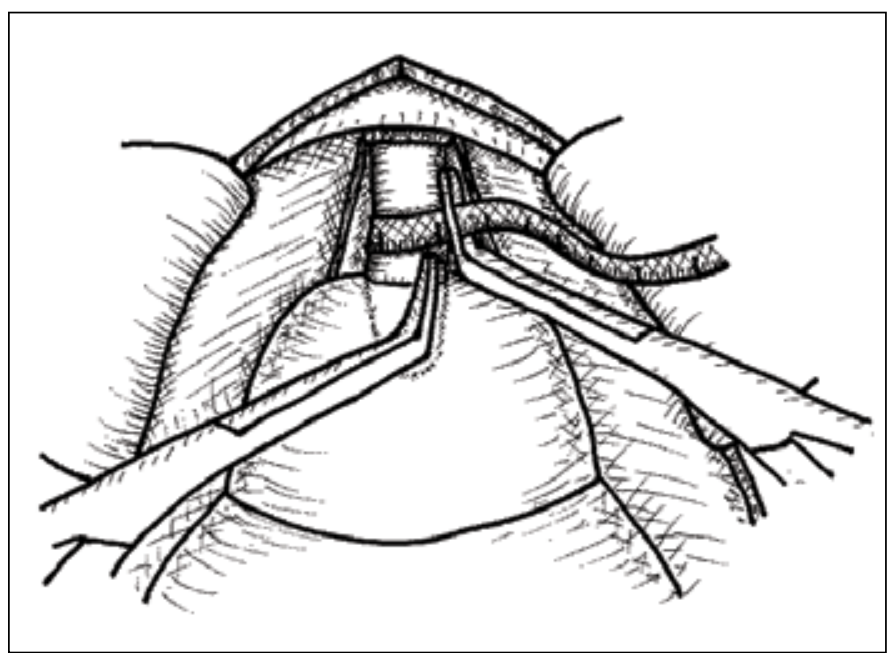

Fig. 3. Illustration of intraoperative measurement of urethral circumference (UC).

\section{Discussion}

Urinary continence after RP has been associated with several variables, including age, prostate volume, prostatic and membranous urethral length, and nerve-sparing status., ${ }^{2,8}$ Cambio and colleagues ${ }^{5}$ reported an exhaustive analysis of the literature identifying the risk factors for urinary incontinence after RP. They analyzed the influence of perioperative factors (body weight, prostate volume, patient age, and pelvic floor exercise), anatomic factors (puboperinealis muscle-sparing dissection, trigonal denervation, and preservation of endopelvic fascia), and technical factors (bladder neck preservation, urethral length preservation, mucosal eversion, neurovascular bundle, and puboprostatic ligament preservation). They concluded that increasing patient age might increase the risk for incontinence after RP and there was no association between increasing patient body weight and prostate volume and worsening continence outcomes. In addition, they insisted that no evidence overwhelmingly supported any surgical technique to improve continence.

Table 1. Clinical and urethral measurement data of 27 patients who underwent radical prostatectomy

\begin{tabular}{lc}
\hline Parameter & Value \\
\hline Age (years) & $66.5 \pm 6.0$ \\
PSA level (ng/mL) & $11.9 \pm 11.6$ \\
Pathologic Gleason score & $7.4 \pm 0.8$ \\
Prostate weight $(\mathrm{g})$ & $39.4 \pm 14.2$ \\
Stretched urethral length (mm) & $24.2 \pm 3.3$ \\
Urethral circumference (mm) & $27.5 \pm 4.4$ \\
MRI urethral length (mm) & $11.3 \pm 1.6$ \\
MRI urethral diameter (mm) & $10.6 \pm 1.9$ \\
Stretched urethral length/MRI urethral length & $2.2 \pm 0.4$ \\
\hline PSA: prostate-specific antigen; MRI: magnetic resonance imaging.
\end{tabular}



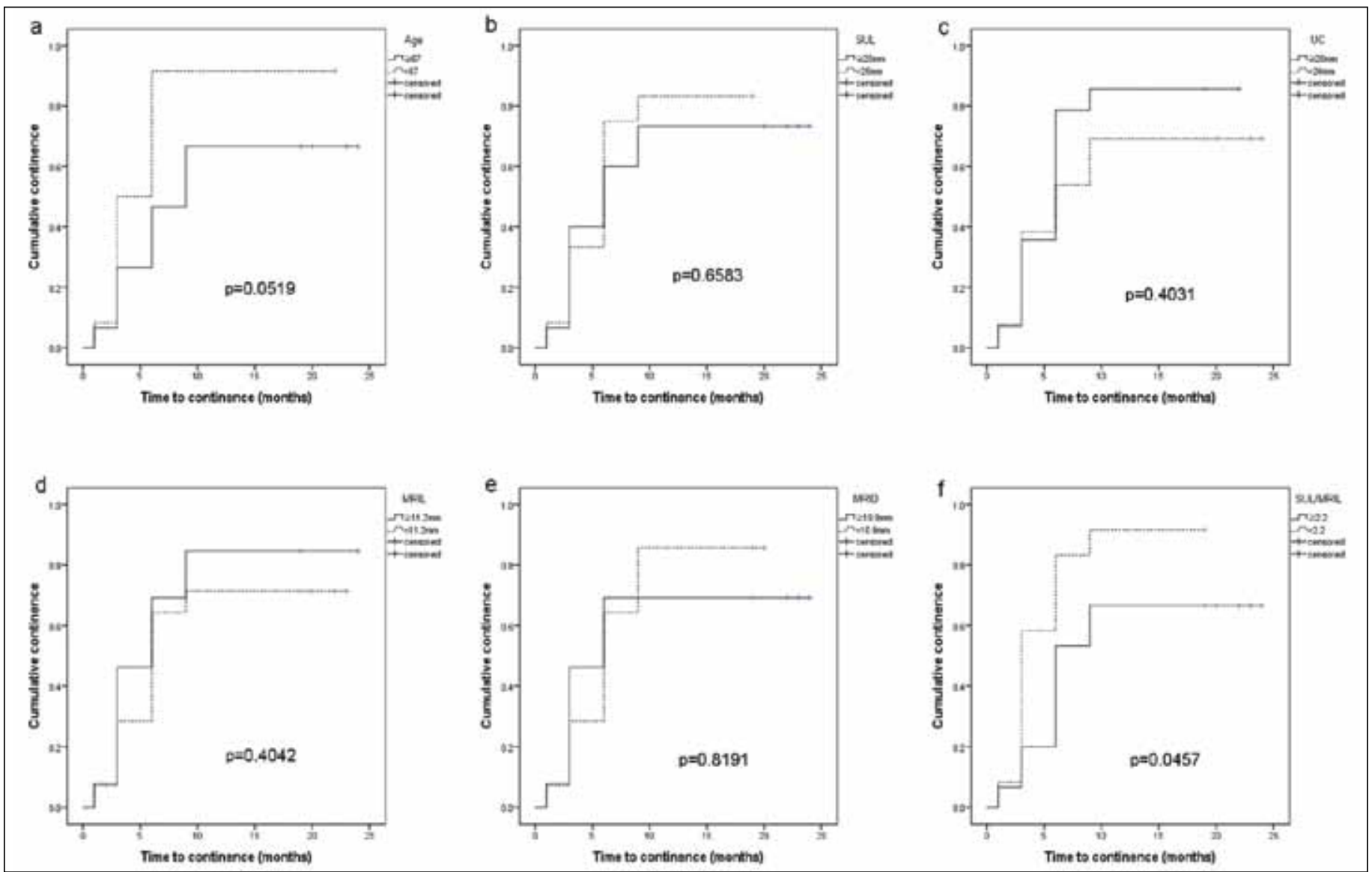

Fig. 4. Kaplan-Meier cumulative continence curve according to age (a), stretched urethral length (b), urethral circumference (c), MRI urethral length (d), magnetic resonance imaging (MRI) urethral diameter (e) and stretched urethral length/MRI urethral length (f).

The improved continence outcomes of longer membranous urethral lengths on preoperative endorectal MRI (eMRI) have been showed by some investigators. Coakley and colleagues ${ }^{8}$ measured preoperative membranous urethral length with eMRI in 180 patients. They found a significant correlation between membranous urethral length and time to stable continence, such that 12 months after RP, $89 \%$ of patients with a membranous urethral length greater than $12 \mathrm{~mm}$ were fully continent, versus only $77 \%$ of patients with a length of $12 \mathrm{~mm}$ or less. As a result, they concluded that membranous urethral length was associated with faster return to stable postoperative continence.

Perhaps the most important factor in the preservation of continence after RP is the preservation of the functional sphincter mechanism or the "intramural distal sphincter mechanism," a term coined by Turner-Warwick. ${ }^{11}$ Here, the sphincteric mechanism comprises the striated sphincter externally and the urethral wall smooth muscle and elastic tissue and the urethral mucosa internally. The striated sphincter is functional only from the prostate apex to the bulb, whereas the internal component of the distal sphincter mechanism extents to the verumontanum. Maximal pres- ervation of this zone that extends from the verumontanum to the penile bulb or perineal membrane is critical in RP. Likewise, Nguyen and colleagues ${ }^{12}$ have reported that their technique of anterior and posterior reconstruction effectively increases the functional urethral length. Their study is based on the relationship between the length of the membranous urethra preoperatively and its positive impact on continence.

To our knowledge, there is only one report on the assessment of intraoperative prostatic and urethral measurements, how they are associated with preoperative eMRI measurements, and whether they can be predictive of continence outcomes. Hakimi and colleagues ${ }^{9}$ analyzed 75 patients who underwent robot-assisted laparoscopic prostatectomy and eMRI by a single surgeon and found that both stretched and cut membranous urethral length correlated with decreased time to continence ( $p=0.03$ and $p=0.04$ respectively) on multivariate analysis. Consequently, they concluded that longer stretched and cut membranous urethral lengths might be associated with faster return to a pad-free state.

Contrary to other studies, we could not demonstrate a significant association between preoperative measurements and continence. This could be partly explained by small 
sample size, but also by innate imprecision of urethral length measurement by eMRI, which gave the impetus for this study. In addition, we demonstrated that membranous urethral length measured during the operation did not affect postoperative continence recovery after RP, which puts the validity of such measurement in question. The insignificance of SUL may derive from two different factors. One may be related to reproducibility of SUL measurement method, which can result from different mechanical traction applied between measurements, inter-individual variability in local anatomy. The other may derive from injury to supporting continence structures. Interestingly, we observed that an excessively long urethra compared to the membranous urethral length on preoperative eMRI is predictive of poorer postoperative continence recovery. It is conceivable that a damaged urethra is more stretchable, leading to a disproportionally lengthy urethra when stretched. A possible cause of damage could be intrinsic or extrinsic during the urethral dissection. Our study suggests that not only the length of the membranous urethra, but also the preservation of its integrity might be important for continence. van Randenborgh and colleagues ${ }^{13}$ analyzed anatomical and functional differences between men with and without postprostatectomy incontinence by urodynamics and MRI. They found that incontinent men had a shorter urethra and were more likely to have distortion of the sphincter area. This result was similar to our study in terms of preservation of membranous urethral integrity.

Our study has several limitations. First, the size of the sample was small, making it difficult to contradict previously suggested association between preoperative MRI measurements and postoperative incontinence. Also, the feasibility and accuracy of intraoperative membranous urethral length and circumference measurement might be questioned. In the early phase of the study, a few cases were excluded because of poor identification of the prostatic apex due to bleeding or an inadvertent early opening of the urethra, leading to an inadequate measurement. However, with added care during the urethral dissection, we were successful with the consecutive cases. In terms of accuracy, we believe that an open intraoperative measurement method such as ours deserves to be the least criticized compared to the radiographic and the endoscopic measurements, because our method is $3 \mathrm{D}$ and utilizes the tactile sense. Lastly, evaluation of pre-, intra- and/or postoperative urethral function using the urodynamic study or the electrical stimulation might have strengthened our results. However, the idea was abandoned due to unavoidable ethical issues.

\section{Conclusion}

Membranous urethral length measured during surgery was not associated with postoperative continence recovery after
RP. Contradictorily, we observed that an excessively long membranous urethra compared to the urethral length on preoperative MRI was predictive of poorer postoperative continence recovery, the significance of which needs be clarified by further studies possibly integrating the evaluation of the functional status of the urethra.

Acknowledgements: This study was supported by a 2009 grant from Ajou University School of Medicine.

Competing interests: The authors declare no competing financial or personal interests.

This paper has been peer-reviewed.

\section{References}

1. Steiner MS, Morton RA, Walsh PC. Impact of anatomical radical prostatectomy on urinary continence. J Urol 1991;145:512-4; discussion 514-5.

2. Eastham JA, Kattan $M W$, Rogers $E$, et al. Risk factors for urinary incontinence after radical prostatectomy. J Urol 1996;156:1707-13. http://dx.doi.org/10.1016/S0022-5347(01)65488-0

3. Majoros $A$, Bach $D$, Keszthelyi $A$, et al. Analysis of risk factors for urinary incontinence after radical prostatectomy. Urol Int 2007;78:202-7. http://dx.doi.org/10.1159/000099338

4. Walsh PC, Donker PJ. Impotence following radical prostatectomy: Insight into etiology and prevention. J Urol 1982;128:492-7.

5. Cambio AJ, Evans (P. Minimising postoperative incontinence following radical prostatectomy: Considerations and evidence. Eur Urol 2006;50:903-13; discussion 513. http://dx.doi.org/10.1016/i. eururo.2006.08.009

6. Takenaka A, Hara R, Soga H, et al. A novel technique for approaching the endopelvic fascia in retropubic radical prostatectomy, based on an anatomical study of fixed and fresh cadavers. BJU Int 2005;95:76671. http://dx.doi.org/10.1111/i.1464-410X.2005.05397.x

7. Song C, Doo CK, Hong JH, et al. Relationship between the integrity of the pelvic floor muscles and early recovery of continence after radical prostatectomy. J Urol 2007;178:208-11. http://dx.doi. org/10.1016/i.juro.2007.03.044

8. Coakley FV, Eberhardt $\mathrm{S}$, Kattan MW, et al. Urinary continence after radical retropubic prostatectomy: Relationship with membranous urethral length on preoperative endorectal magnetic resonance imaging. J Urol 2002; 168:1032-5.

9. Hakimi AA, Faleck DM, Agalliu I, et al. Preoperative and intraoperative measurements of urethral length as predictors of continence after robot-assisted radical prostatectomy. J Endourol 201 1;25:1025-30. http://dx.doi.org/10.1089/end.2010.0692

10. Paparel P, Akin 0, Sandhu IS, et al. Recovery of urinary continence after radical prostatectomy: Association with urethral length and urethral fibrosis measured by preoperative and postoperative endorectal magnetic resonance imaging. Eur Urol 2009;55:629-37. http://dx.doi.org/10.1016/i.eururo.2008.08.057

11. Warwick RT, Whiteside CG, Arnold EP, et al. A urodynamic view of prostatic obstruction and the results of prostatectomy. Br J Urol 1973;45:631-45. http://dx.doi.org/10.1111/i.1464-410X.1973.tb12234.x

12. Nguyen L, Jhaveri J, Tewari A. Surgical technique to overcome anatomical shortcoming: Balancing postprostatectomy continence outcomes of urethral sphincter lengths on preoperative magnetic resonance imaging. J Urol 2008;179:1907-11. http://dx.doi.org/10.1016/i.juro.2008.01.036

13. van Randenborgh H, Paul R, Kubler H, et al. Improved urinary continence after radical retropubic prostatectomy with preparation of a long, partially intraprostatic portion of the membraneous urethra: An analysis of 1013 consecutive cases. Prostate Cancer Prostatic Dis 2004;7:253-7.

Correspondence: Dr. Sun II Kim, Department of Urology, Ajou University School of Medicine, Yeongtong-gu, Suwon-si, Gyeonggi-do, South Korea; sikimuro@ajou.ac.kr 